\title{
The loaded parabolic-hyperbolic equation and its relation to non-local problems
}

\author{
U. I. Baltaeva \\ National University of Uzbekistan named after Mirzo Ulugbek \\ Differential equations and Mathematical physics, 100174, Tashkent, Uzbekistan \\ umida_baltayeva@mail.ru
}

DOI 10.17586/2220-8054-2017-8-4-413-419

In this present paper, unique solvability is proved for the boundary value problems for the loaded differential equations associated with non-local boundary value problems, for the classical partial differential equations.

Keywords: parabolic-hyperbolic type equations, non-local problems, loaded equation, boundary value problems, gluing condition, integral equation.

Received: 25 July 2017

Revised: 3 August 2017

\section{Introduction}

Research on equations of parabolic-hyperbolic and hyperbolic-elliptic types has been conducted heavily. Since, there are both theoretical and practical uses for their applications, especially in the development of principal parts of partial differential equations. Moreover, in recent years it has become increasingly important to investigate a new class of equations, known as loaded equations, as a direct result of issues with the optimal control of the agro-economical system, long-term forecasting and regulation of groundwater levels and soil moisture [1-4].

Basic questions on the theory of boundary value problems for partial differential equations still exist, and are equivalent to that of the boundary value problems for loaded differential equations. However, the existence of the loaded part operator A does not often make it possible to directly apply the known theory of boundary value problems, for the classical partial differential equations. In view of this, searching for the solutions of loaded differential equation using preassigned classes, it may be possible to reduce to them to new problems, for non-loaded equations(see [4-6]).

At the same time, mixed type equations appear in some problems of nanophysics and are present in some micro- and nanoflow models. Particularly, parabolic-hyperbolic equations are considered in some models of spinodal decomposition (so-called hyperbolic diffusion, see, e.g. [7,8]), and also described flow in thin viscous layers subjected to ultrasonic acoustic field [9].

The present work is devoted to the formulation and investigation of the boundary value problems for a loaded equation of the mixed parabolic hyperbolic type:

$$
\frac{\partial}{\partial x}(L u)=\mu \sum_{i=1}^{n} a_{i}(x) D_{0 x}^{\alpha_{i}}\left[u_{y}(x, 0)-u(x, 0)\right],
$$

where

$$
\begin{gathered}
L u \equiv u_{x x}-\frac{1-\operatorname{sgn} y}{2} u_{y y}-\frac{1+\operatorname{sgn} y}{2} u_{y}-\lambda u, \\
D_{0 x}^{\alpha_{i}} f(x)= \begin{cases}\frac{1}{\Gamma\left(-\alpha_{i}\right)} \int_{0}^{x} \frac{f(t) d t}{(x-t)^{1+\alpha_{i}}}, & \alpha_{i}<0, \\
\frac{d}{d x} D_{0 x}^{\alpha_{i}-1} f(x), & 0<\alpha_{i}<1,\end{cases}
\end{gathered}
$$

integro-differential operator [4]. $D_{0 x}^{-\alpha_{i}} D_{0 x}^{\alpha_{i}} f \equiv D_{0 x}^{0} f \equiv f(x)$. We assume, that $\alpha_{n}<\alpha_{n-1}<\ldots<\alpha_{1}=\alpha<1$ and coefficients $a_{i}=a_{i}(x) \in C^{1}[0,1] \cap C^{3}(0,1), \lambda, \mu$ are given real parameters, and $\lambda>0$.

Furthermore, we will discuss how the boundary value problem for the loaded differential equation is associated with non-local boundary value problems, for classical partial differential equations. In our investigations, we have formulated first-kind boundary value problems (such as the Tricomi problems) for the linearly loaded, integrodifferential equation of the third order, with mixed operators. 


\section{First kind of boundary value problems for a loaded equation of the mixed type}

Let $\Omega_{1} \subset R^{2}$ be a domain bounded by the segments $A B, B B_{0}, A A_{0}, A_{0} B_{0}$ of straight lines $y=0, x=1$, $x=0, y=h$, respectively when $y>0 . \Omega_{2}$ is a characteristic triangle bounded by the segment $A B$ the axis $O X$ and two characteristics:

of equation (1) for $y<0$.

$$
A C: x+y=0, \quad B C: x-y=1,
$$

Let us introduce the following notation:

$$
I=\{(x, y): 0<x<1, y=0\}, \quad \Omega=\Omega_{1} \cup \Omega_{2} \cup I .
$$

Problem 2.1. Find a regular solution $u(x, y)$ equation (1) in $\Omega$, which has continuous derivatives $u_{x}\left(u_{y}\right)$, up to $A A_{0} \cup A B \cup A C(A B \cup A C)$, and satisfies the boundary value conditions:

$$
\begin{gathered}
u(0, y)=\varphi_{1}(y), \quad u(1, y)=\varphi_{2}(y), u_{x}(0, y)=\varphi_{3}(y), 0 \leq y \leq h, \\
u(x,-x)=\psi_{1}(x),\left.\quad \frac{\partial u(x, y)}{\partial n}\right|_{y=-x}=\psi_{2}(x), \quad 0 \leq x \leq \frac{1}{2},
\end{gathered}
$$

and the gluing conditions:

$$
u_{y}(x,+0)=u_{y}(x,-0), \quad(x, 0) \in I,
$$

where $\varphi_{1}(y), \varphi_{2}(y), \varphi_{3}(y), \psi_{1}(x)$ and $\psi_{2}(x)$ are the given functions.

Problem 2.2. Find a regular solution $u(x, y)$ equation (1) in $\Omega$, which has continuous derivatives $u_{x}\left(u_{y}\right)$, up to $A A_{0} \cup A B \cup B C(A B \cup B C)$, and satisfies the boundary value conditions (2),

$$
u(x, x-1)=\tilde{\psi}_{1}(x),\left.\quad \frac{\partial u(x, y)}{\partial n}\right|_{y=x-1}=\tilde{\psi}_{2}(x), \quad \frac{1}{2} \leq x \leq 1,
$$

and the gluing condition:

$$
u_{y}(x,+0)=u_{y}(x,-0), \quad(x, 0) \in I,
$$

where $\tilde{\psi}_{1}(x)$ and $\tilde{\psi}_{2}(x)$ are the given functions.

We note that physical characteristic problems may reduce to equations which include, some composed operator in the loaded part. The unique solvability of the analogs of the Tricomi for the loaded differential equations was investigated in $[5,10]$, for which the loaded part contains the imputation or imputation operators of the unknown function.

An important aspect of the investigation of the boundary value problems, is shown by the following theorem. The following theorem holds.

Theorem 2.1. Any regular solution of equation (1) (when $y \neq 0)$ is represented in the form:

where $z(x, y)$ is a solution to the equation:

$$
u(x, y)=z(x, y)+w(x),
$$

$$
\frac{\partial}{\partial x}\left(z_{x x}-\frac{1-\operatorname{sgn} y}{2} z_{y y}-\frac{1+\operatorname{sgn} y}{2} z_{y}-\lambda z\right)=0,
$$

$w(x)$ is a solution of the following ordinary differential equation:

$$
w^{\prime \prime \prime}(x)-\lambda w^{\prime}(x)-\mu \sum_{i=1}^{n} a_{i} D_{0 x}^{\alpha_{i}(x)} w(x)=\mu \sum_{i=1}^{n} a_{i}(x) D_{0 x}^{\alpha_{i}}\left[z_{y}(x, 0)-z(x, 0)\right] .
$$

Proof of Theorem 2.1.

Let $u(x, y)$, represented by formula (5), be the solution of equation (1) for $y<0$. Then, substituting:

$$
\begin{aligned}
& \frac{\partial}{\partial x}\left(u_{x x}-u_{y y}-\lambda u\right)-\mu \sum_{i=1}^{n} a_{i}(x) D_{0 x}^{\alpha_{i}}\left[u_{y}(x, 0)-u(x, 0)\right]=\frac{\partial}{\partial x}\left(z_{x x}-z_{y y}-\lambda z\right)+ \\
& +w^{\prime \prime \prime}(x)-\lambda w^{\prime}(x)-\mu \sum_{i=1}^{n} a_{i}(x) D_{0 x}^{\alpha_{i}} w(x)-\mu \sum_{i=1}^{n} a_{i}(x) D_{0 x}^{\alpha_{i}}\left[z_{y}(x, 0)-z(x, 0)\right]=0,
\end{aligned}
$$

satisfies equation (1) for $y<0$. 
Then, vice versa, let $u(x, y)$ be a regular solution to equation (1), and $w(x)$ be a certain solution:

$$
w^{\prime \prime \prime}(x)-\lambda w^{\prime}(x)-\mu \sum_{i=1}^{n} a_{i}(x) D_{0 x}^{\alpha_{i}} w(x)=\mu \sum_{i=1}^{n} a_{i}(x) D_{0 x}^{\alpha_{i}}\left[u_{y}(x, 0)-u(x, 0)\right] .
$$

Let us prove the validity of the relation (5). Manifestly, the function:

$$
u(x, y)=z(x, y)+\frac{\mu}{\lambda} \int_{0}^{x}(\cosh \sqrt{\lambda}(x-t)-1) \sum_{i=1}^{n} a_{i}(x) D_{0 t}^{\alpha_{i}}\left[u_{y}(t, 0)-u(t, 0)\right] d t,
$$

is a solution to equation (1), where $z(x, y)$ is a solution to equation (6), and the function:

$$
u(x, y)=-\frac{\mu}{\lambda} \int_{0}^{x}(1-\cosh \sqrt{\lambda}(x-t)) \sum_{i=1}^{n} a_{i}(t) D_{0 t}^{\alpha_{i}}\left[u_{y}(t, 0)-u(t, 0)\right] d t,
$$

is a partial solution to equation (1). Hence, (1) highlights the validity of the representation (5), i.e. $u(x, y)=z(x, y)+w(x)$.

It follows from the latter representation that $u(x, 0)=z(x, 0)+w(x)$. Then, (8) provides:

$$
w^{\prime \prime \prime}(x)-\lambda w^{\prime}(x)-\mu \sum_{i=1}^{n} a_{i}(x) D_{0 x}^{\alpha_{i}} w(x)-\mu \sum_{i=1}^{n} a_{i}(x) D_{0 x}^{\alpha_{i}}\left[z_{y}(x, 0)-z(x, 0)\right]=0,
$$

and the function $z(x, y)=u(x, y)-w(x)$, satisfies equation (6) for $y<0$.

Analogously proved in the case for $y<0$. Theorem 2.1 is proved.

Now, invoking that the function $a e^{\sqrt{\lambda} x}+b e^{-\sqrt{\lambda} x}+c$ satisfies equation (6), we can assume without loss of generality that:

$$
w(0)=w^{\prime}(0)=w^{\prime \prime}(0)=0,
$$

when studying Problem 2.1 and Problem 2.2.

Let us solve the Cauchy problem for equation (7) with the conditions (9), with respect to $w(x)$. Assuming that:

we write equation (7) in the form:

$$
\widetilde{f}(x)=\mu \sum_{i=1}^{n} a_{i}(x) D_{0 x}^{\alpha_{i}} w(x)+\mu \sum_{i=1}^{n} a_{i}(x) D_{0 x}^{\alpha_{i}}\left[z_{y}(x, 0)-z(x, 0)\right],
$$

$$
\omega(x)=\frac{1}{\lambda} \int_{0}^{x}(\cosh \sqrt{\lambda}(x-t)-1) \tilde{f}(t) d t .
$$

The last equality with respect to designation and after some transformation becomes:

$$
\omega(x)-\mu \int_{0}^{x} K(x, t) \omega(t) d t=F(x), \quad 0 \leq x \leq 1,
$$

where

$$
\begin{gathered}
K(x, t)=\frac{1}{\Gamma\left(-\alpha_{i}\right)} \int_{0}^{x} A_{i}(t)(t-s)^{-1-\alpha_{i}}(\cosh \sqrt{\lambda}(x-t)-1) d t, \quad \alpha_{i}<0, \\
K(x, t)=\frac{1}{\lambda \Gamma\left(1-\alpha_{i}\right)} \int_{s}^{x} \frac{\partial}{\partial t}\left[A_{i}(t)(t-s)^{-\alpha_{i}}(\cosh \sqrt{\lambda}(x-t)-1)\right] d t, 0<\alpha_{i}<1, \\
F(x)=\frac{\mu}{\lambda} \int_{0}^{x}(\cosh \sqrt{\lambda}(x-t)-1) \sum_{i=1}^{n} a_{i}(t) D_{0 x}^{\alpha_{i}}\left[z_{y}(t, 0)-z(t, 0)\right] d t .
\end{gathered}
$$

The recurring index $i=1,2, \ldots n$, implies summation. Therefore, solving the next equation with respect to [11], we have a solution to the Cauchy problem for equation (7) with the conditions (9):

$$
\omega(x)=\int_{0}^{x} P(x, t) \sum_{i=1}^{n} a_{i}(t) D_{0 x}^{\alpha_{i}}\left[z_{y}(t, 0)-z(t, 0)\right] d t,
$$


where

$$
P(x, t)=\frac{\mu}{\lambda}\left\{\cosh \sqrt{\lambda}(x-t)-1+\mu \int_{t}^{x}(\cosh \sqrt{\lambda}(s-t)-1) R(x, s ; \mu) d s\right\} .
$$

$R(x, s ; \mu)$ is the resolvent of the kernel $K(x, t)$, when studying Problem 2.1 and Problem 2.2. Thus, by virtue of the representation (5), equation (1) Problem 2.1 and Problem 2.2, in view of (9), are all reduced to the following Problem $N P_{1}$ and Problem $N P_{2}$ for equation (6).

We investigate the following problems:

Problem $N \boldsymbol{P}_{1}$. To find a regular solution to equation (6) in the domains $\Omega_{1}$ and $\Omega_{2}$, which has continuous derivatives $u_{x}\left(u_{y}\right)$, up to $A A_{0} \cup A C(A C)$, and satisfies the boundary conditions:

$$
\begin{gathered}
z(0, y)=\varphi_{1}(y), z(0, y)=\varphi_{2}(y)-w(1), \quad 0 \leq y \leq h, \\
z_{x}(0, y)=\varphi_{3}(y), \quad 0 \leq y \leq h, \\
z(x,-x)=\psi_{1}(x)-\int_{0}^{x} P(x, t) \sum_{i=1}^{n} a_{i}(t) D_{0 x}^{\alpha_{i}}\left[z_{y}(t, 0)-z(t, 0)\right] d t, 0 \leq x \leq \frac{1}{2}, \\
\frac{\partial z(x,-x)}{\partial n}=\psi_{2}(x)-\frac{1}{\sqrt{2}} \int_{0}^{x} P_{x}^{\prime}(x, t) \sum_{i=1}^{n} a_{i}(t) D_{0 x}^{\alpha_{i}}\left[z_{y}(t, 0)-z(t, 0)\right] d t, 0 \leq x \leq \frac{1}{2},
\end{gathered}
$$

and the gluing condition:

$$
z_{y}(x,+0)=z_{y}(x,-0),(x, 0) \in I,
$$

where $\varphi_{1}(y), \varphi_{2}(y), \varphi_{3}(y), \psi_{1}(x)$ and $\psi_{2}(x)$ are given functions, such that $\varphi_{1}(0)=\psi_{1}(0)$.

Problem $N \boldsymbol{P}_{2}$. To find a regular solution of equation (6) in the domains $\Omega_{1}$ and $\Omega_{2}$, has continuous derivatives $u_{x}\left(u_{y}\right)$, up to $A A_{0} \cup B C(B C)$, and satisfies the boundary conditions (16), (17),

$$
\begin{gathered}
z(x, x-1)=\tilde{\psi}_{1}(x)-\int_{0}^{x} P(x, t) \sum_{i=1}^{n} a_{i}(t) D_{0 x}^{\alpha_{i}}\left[z_{y}(t, 0)-z(t, 0)\right] d t, \frac{1}{2} \leq x \leq 1, \\
\frac{\partial z(x, x-1)}{\partial n}=\tilde{\psi}_{2}(x)-\frac{1}{\sqrt{2}} \int_{0}^{x} P_{x}^{\prime}(x, t) \sum_{i=1}^{n} a_{i}(t) D_{0 x}^{\alpha_{i}}\left[z_{y}(t, 0)-z(t, 0)\right] d t, \frac{1}{2} \leq x \leq 1,
\end{gathered}
$$

and the gluing condition:

$$
z_{y}(x,+0)=z_{y}(x,-0),(x, 0) \in I,
$$

where $\tilde{\psi}_{1}(x)$ and $\tilde{\psi}_{2}(x)$ are given functions, such that $\varphi_{2}(0)=\tilde{\psi}_{1}(1)$.

Since Problem 2.1 and Problem 2.2 were reduced to the equivalent non-local Problems $N P_{1}$ and $N P_{2}$ for the third order mixed type equation (6), we may conclude that Problems 2.1 and 2.2 have a unique solutions, as a direct result of the unique solvability of the non-local problem. The early 1970's marked the beginning of the systematic study of the third and higher order, mixed and mixed composite type PDEs; which contain parabolic-hyperbolic, hyperbolic-elliptic and elliptic-parabolic operators in the main part. This subject has been studied extensively and has been developed by numerous mathematicians [12-14], due to, the increasing popularity of its connection with non-local problems, and the appearance of these PDEs in mathematical models for various problems which possess physical traits. For instance, while studying problems of moisture transfer in soils, heat transfer in heterogeneous media, diffusion of thermal neutrons in inhibitors, simulation of various biological processes, phenomena etc [6,15-18].

The following theorems hold.

Theorem 2.2. If $\lambda>0$ and $\varphi_{1}(0)=\psi_{1}(0)$,

$$
\begin{gathered}
\varphi_{j}(y) \in C^{1}[0, h],(j=1,2), \varphi_{3}(y) \in C[0, h] \cap C^{1}(0, h), \\
\psi_{1}(x) \in C^{1}[0,1 / 2] \cap C^{3}(0,1 / 2), \psi_{2}(x) \in C[0,1 / 2] \cap C^{2}(0,1 / 2),
\end{gathered}
$$

then there exists a unique solution to the Problem 2.1 in the domain $\Omega$. 
Theorem 2.3. If $\lambda>0$ and $\varphi_{2}(0)=\tilde{\psi}_{1}(1)$,

$$
\begin{gathered}
\varphi_{j}(y) \in C^{1}[0, h],(j=1,2), \varphi_{3}(y) \in C[0, h] \cap C^{1}(0, h), \\
\tilde{\psi}_{1}(x) \in C^{1}[1 / 2,1] \cap C^{3}(1 / 2,1), \tilde{\psi}_{2}(x) \in C[1 / 2,1] \cap C^{2}(1 / 2,1),
\end{gathered}
$$

then there exists a unique solution to the Problem 2.2 in the domain $\Omega$.

\section{Existence of solution to Problem 2.1}

Let us introduce the notation:

$$
u(x, \pm 0)=\tau(x), u_{y}(x, \pm 0)=\nu(x),
$$

now with regards (5) and gluing conditions, we get:

$$
\begin{gathered}
z(x, \pm 0)=\tau(x)-\omega(x), \quad 0 \leq x \leq 1 \\
\left.\frac{\partial z(x, y)}{\partial y}\right|_{y= \pm 0}=\nu(x), \quad 0<x<1
\end{gathered}
$$

A solution of the Darboux problem for the equation (6) with the boundary value conditions (18), (19) and (22) in the domain $\Omega_{2}$ can be represented as:

$$
\begin{gathered}
z(x, y)=\tau^{*}(x+y)+\psi_{1}^{*}\left(\frac{x-y}{2}\right)-\psi_{1}^{*}\left(\frac{x+y}{2}\right)- \\
-\lambda y \int_{0}^{x+y} \tau^{*}(t) \bar{I}_{1}[\sqrt{\lambda(x-y-t)(x+y-t)}] d t+\sqrt{\lambda} \int_{0}^{y} \sin \sqrt{\lambda}(y-t) \psi_{1}^{*}(-t) d t+ \\
+\sqrt{2} \int_{0}^{y} \cos \sqrt{\lambda}(y-t) \psi_{2}^{*}(-t) d t+\lambda \int_{0}^{\frac{x-y}{2}} g_{1}(x-y, x+y, t) \psi_{1}^{*}(t) d t- \\
-\lambda \int_{0}^{\frac{x+y}{2}} g_{1}(x+y, x-y, t) \psi_{1}^{*}(t) d t+\sqrt{2} \int_{0}^{\frac{x-y}{2}} g_{2}(x-y, x+y, t) \psi_{2}^{*}(t) d t- \\
-\sqrt{2} \int_{0}^{\frac{x-y}{2}} g_{2}(x+y, x-y, t) \psi_{2}^{*}(t) d t-\sqrt{\frac{2}{\lambda}} \psi_{2}^{*}(0)\left\{\sin \sqrt{\lambda}\left(\frac{x-y}{2}\right)-\right. \\
-\sin \sqrt{\lambda}\left(\frac{x+y}{2}\right)+\sin \sqrt{\lambda} y+\lambda(x+y) \int_{0}^{\frac{x-y}{2}} \bar{I}_{1}[\sqrt{\lambda(x+y)(x-y-2 t)}] \sin \sqrt{\lambda} t d t- \\
\left.-\lambda(x-y) \int_{0}^{\frac{x+y}{2}} \bar{I}_{1}[\sqrt{\lambda(x-y)(x+y-2 t)}] \sin \sqrt{\lambda} t d t\right\},
\end{gathered}
$$

where

$$
\begin{gathered}
g_{1}(x, y, t)=\frac{1}{\sqrt{\lambda}} \sin \sqrt{\lambda}\left(t-\frac{x}{2}\right)+y \bar{I}_{1}[\sqrt{\lambda y(x-2 t)}]+\sqrt{\lambda} y \int_{t}^{x / 2} \sin \sqrt{\lambda}(t-s) \bar{I}_{1}[\sqrt{\lambda y(x-2 s)}] d s \\
g_{2}(x, y, t)=\cos \sqrt{\lambda}\left(t-\frac{x}{2}\right)+\lambda y \int_{t}^{x / 2} \cos \sqrt{\lambda}(t-s) \bar{I}_{1}[\sqrt{\lambda y(x-2 s)}] d s \\
\psi_{1}^{*}(x)=\psi_{1}(x)-\tilde{w}(x), \quad \psi_{2}^{*}(x)=\psi_{2}(x)-\frac{1}{\sqrt{2}} \tilde{w}^{\prime}(x) \\
\tilde{w}(x)=\frac{\mu}{\lambda} \int_{0}^{x}(x)=\tau(x)-\tilde{w}(x)
\end{gathered}
$$


$\bar{I}_{1}(x)=I_{1}(x) / x, I_{1}(x)$ is the modified Bessel function (Bessel function of the first kind with imaginary argument [19]).

Differentiating (24) with respect to $y$ and passing through the limit $y \rightarrow+0$ with the preceding notations and (23), we obtain first functional relation between the function $\tau(x)$ and $\nu(x)$, transferred from the $\Omega_{1}$ to $A B$ :

$$
\begin{gathered}
\nu(x)+\mu \int_{0}^{x} L_{1}(x, t) \sum_{i=1}^{n} a_{i} D_{0 t}^{\alpha_{i}}[\nu(t)-\tau(t)] d t- \\
-\mu \int_{0}^{x / 2} L_{2}(x, t) \sum_{i=1}^{n} a_{i} D_{0 t}^{\alpha_{i}}[\nu(t)-\tau(t)] d t=\tau^{\prime}(x)-\lambda \int_{0}^{x} \tau(t) \bar{I}_{1}[\sqrt{\lambda}(x-t)] d t+f(x),
\end{gathered}
$$

where

$$
L_{1}(x, t)=\frac{1}{\sqrt{\lambda}} \sinh \sqrt{\lambda}(x-t)+\int_{t}^{x}(1-\cosh \sqrt{\lambda}(s-t)) \bar{I}_{1}[\sqrt{\lambda}(x-s)] d s,
$$

$L_{2}(x, t), f(x)$ are continuously differentiable functions.

Further, from (6) considering the property of Problem 2.1, (22) and (23), passing through the limit we obtain:

$$
\tau^{\prime \prime}(x)-\lambda \tau(x)=k+\nu(x)+w^{\prime \prime}(x)-\lambda w(x),
$$

where $k$ is an unknown constant to be defined.

The equality (28) is a second functional relation between $\tau(x)$ and $\nu(x)$, transferred from the domain $\Omega_{1}$ to $A B$.

Solving equation (28) with respect to $\tau(x)$ with the conditions:

we have

$$
\tau(0)=\varphi_{1}(0), \quad \tau^{\prime}(0)=\varphi_{3}(0),
$$

$$
\begin{gathered}
\tau(x)=\int_{0}^{x} \frac{\sinh \sqrt{\lambda}(x-t)}{\sqrt{\lambda}} \nu(t) d t-\frac{\mu}{\lambda} \int_{0}^{x}(1-\cosh \sqrt{\lambda}(x-t)) \sum_{i=1}^{n} a_{i} D_{o t}^{\alpha_{i}}[\nu(t)-\tau(t)] d t- \\
\frac{k}{\lambda}(1-\cosh \sqrt{\lambda} x)+\varphi_{1}(0) \cosh \sqrt{\lambda} x+\frac{1}{\sqrt{\lambda}} \varphi_{3}(0) \sinh \sqrt{\lambda} x .
\end{gathered}
$$

Thus, we get (27) and (30) system of the equations for the functions $\tau(x)$ and $\nu(x)$. If we write relation (30), in the form

$$
\begin{gathered}
\tau(x)-\frac{\mu}{\lambda} \int_{0}^{x}(1-\cosh \sqrt{\lambda}(x-t)) \sum_{i=1}^{n} a_{i} D_{o t}^{\alpha_{i}} \tau(t) d t=\int_{0}^{x} \frac{\sinh \sqrt{\lambda}(x-t)}{\sqrt{\lambda}} \nu(t) d t- \\
\frac{\mu}{\lambda} \int_{0}^{x}(1-\cosh \sqrt{\lambda}(x-t)) \sum_{i=1}^{n} a_{i} D_{o t}^{\alpha_{i}} \nu(t) d t- \\
\frac{k}{\lambda}(1-\cosh \sqrt{\lambda} x)+\varphi_{1}(0) \cosh \sqrt{\lambda} x+\frac{1}{\sqrt{\lambda}} \varphi_{3}(0) \sinh \sqrt{\lambda} x
\end{gathered}
$$

and assuming that:

$$
\begin{gathered}
\Phi(x)=\int_{0}^{x} \frac{\sinh \sqrt{\lambda}(x-t)}{\sqrt{\lambda}} \nu(t) d t-\frac{\mu}{\lambda} \int_{0}^{x}(1-\cosh \sqrt{\lambda}(x-t)) \sum_{i=1}^{n} a_{i} D_{o t}^{\alpha_{i}} \nu(t) d t- \\
\frac{k}{\lambda}(1-\cosh \sqrt{\lambda} x)+\varphi_{1}(0) \cosh \sqrt{\lambda} x+\frac{1}{\sqrt{\lambda}} \varphi_{3}(0) \sinh \sqrt{\lambda} x,
\end{gathered}
$$

for $\alpha_{i}<0$ and $0<\alpha_{i}<1$ applying the Dirichlet permutation integration formula and corresponding to change of variables $s=t+(x-t) v$, we obtain the second kind Volterra type integral equation:

$$
\tau(x)-\mu \int_{0}^{x} \widetilde{K}(x, t) \tau(t) d t=\Phi(x),
$$


where

$$
\widetilde{K}(x, t)=-\frac{(x-t)^{-\alpha_{i}}}{\lambda \Gamma\left(-\alpha_{i}\right)} \int_{0}^{1} \frac{A_{i}\left(1-\cosh \left[\sqrt{\lambda_{2}}(x-t)(1+v)\right]\right)}{v^{1+\alpha_{i}}} d v, \text { at } \alpha_{i}<0,
$$

and at $0<\alpha_{i}<1$

$$
\widetilde{K}(x, t)=\frac{(x-t)^{1-\alpha_{i}}}{\lambda \Gamma\left(1-\alpha_{i}\right)} \int_{0}^{1} v^{-\alpha_{i}} \frac{\partial}{\partial v}\left[A_{i}\left(1-\cosh \left[\sqrt{\lambda_{2}}(x-t)(1+v)\right]\right)\right] d v .
$$

From the representations of the functions $\widetilde{K}(x, t)$ and $\Phi(x)$, based on the general theory of integral equations [11], one can be certain that (33) has a unique solution, which is represented as:

$$
\tau(x)=\Phi(x)+\int_{0}^{x} \widetilde{R}(x, t) \Phi(t) d t, \quad 0 \leq x \leq 1,
$$

where $\widetilde{R}(x, t)$ is the resolvent of the kernel $\widetilde{K}(x, t)$. Substituting (34) in (27), consider (32) and the sewing condition, we may obtain the integral equation with a a shift, with respect to $\nu(x)$, by which a kernel exists with weak singularity. Therefore, by using successive approximations [11], with regards to the conditions of the theorem and (32), we can uniquely define the function $\nu(x)$ at $\lambda \neq 0, a_{i}=a_{i}(x) \in C[0,1] \cap C^{3}(0,1)$. Upon determining $\tau(x)$ and $\nu(x)$, we find the functions $w(x)$ from (14). Hence, by virtue of the condition $\tau(1)=\varphi_{2}(0)-w(1), k$ are determined uniquely.

After finding $\tau(x)$ and $\nu(x)$, the solution of the Problem $N P_{1}$ defined in $\Omega_{2}$ by formula Cauchy-Goursat (24) or Cauchy in [19] and in the domain $\Omega_{1}$ we arrive to the problem for a non loaded equation of the third order [12].

Thus, we now know all particulars, such that the solution of Problem 2.1, in the domains $\Omega_{1}$ and $\Omega_{2}$ can be constructed from (5).

Problem 2.1 is uniquely solvable. Thus, Theorem 2.2 is proved. Analogously, one can also prove the uniqueness of the solution to Problem 2.2.

\section{References}

[1] Nakhushev A.M. Loaded equations and their applications, Differential equations, 1983, 19(1), P. 86-94.

[2] Wiener J., Debnath L. Partial differential equations with piecewise constant delay, Internat. J. Math. and Math. Scz., 1991, 14, P. 485-496.

[3] Dzhenaliev M.T. A remark on the theory of linear boundary value problems for loaded differential equations. Almaty, Institute of Theoretical and Applied Mathematics, 1995, $270 \mathrm{p}$.

[4] Nakhushev A.M. Equations of mathematical biology. Vishaya shkola, Moscow, 1995, $302 \mathrm{p}$.

[5] Baltaeva U.I. Solvability of the analogs of the problem Tricomi for the mixed type loaded equations with parabolic-hyperbolic operators. Boundary Value Problems, 2014, 21, P. 1-12.

[6] Nakhushev A.M. Nonlocal problem and the Goursat problem for loaded hyperbolic equation and their application in prediction of ground moisture. Soviet Math. Dokl., 1978, 19(5), P. 1243-1247.

[7] Kirillova S.A., Almjashev V.I., Gusarov V.V. Spinodal decomposition in the $\mathrm{SiO}_{2}-\mathrm{TiO}_{2}$ system and hierarchically organized nanostructures formation. Nanosystems: Phys. Chem. Math., 2012, 3(2), P. 100-115.

[8] Antonov N.M., Popov I.Yu., Gusarov V.V. Model of spinodal decomposition of phases under hyperbolic diffusion. Physics of the Solid State, 1999, 41(5), P. 824-826.

[9] Melikhov I.F. Asymptotic solution of ultrasonic near-field levitation problem. Nanosystems: Phys. Chem. Math., 2017, 8(2), P. 260-265.

[10] Islomov B.I., Baltaeva U.I. Boundary value problems for the loaded equations of the hyperbolic and mixed types. Ufa mathematical journal, 2011, 3(3), P. 15-25.

[11] Krasnov M.L. Integral equations. Introduction to the theory. Moscow, Nauka, 1975

[12] Dzhurayev T.D., Sopuev A. and Mamayhonov M. Boundary value problems for the problems for the parabolic-hyperbolic type equations. Tashkent, Fan, 1986.

[13] Sabytov K.B. To the theory of mixed parabolic-hyperbolic type equations with a spectral parameter, Differential equations, 1989, 25(1), P. 117-126.

[14] Salakhitdinov M.S., Urinov A.K. Boundary value problems for equations of mixed type with a spectral parameter. Tashkent, Fan, 1997.

[15] Berdyshev A.S. Non-local boundary value problems for the mixed type equation in a domain with deviation from a characteristic. Differential equations, 1993, 29(12), P. 2118-2125.

[16] Serbina L.I. Nonlocal mathematical models of transport processes in systems with a fractal structure. Nal'chik, $2002,144 \mathrm{p}$.

[17] Berdyshev A.S., Karimov E.T. Some non-local problems for the parabolic-hyperbolic type equation with non-characteristic line of changing type. Differential equations, 2006, 4(2), P. 183-193.

[18] Kishin B. Sadarangani, Abdullaev Obidjon Kh. About a problem for loaded parabolic-hyperbolic type equation with fractional derivatives. International Journal of Differential equations, Volume 2016, Article ID 9815796, 6 p.

[19] Tikhonov A.N., Samarskij A.A. Equations of Mathematical Physics. Nauka, Moscow, 1966. 